\title{
Erwerbstätigkeit, -minderung und -unfähigkeit, Pfade in die Rente
}

\section{Bellmann}

\section{Einleitung}

Seinen letzten Artikel als Mitglied der Wirtschaftsredaktion der "Zeit“ hat Dietmar Lamparter der Diskriminierung von Arbeitnehmer*innen jenseits der 50 gewidmet: „Viele Unternehmen unterschätzen die Fähigkeiten älterer Mitarbeiter - dabei fehlen Fachkräfte." (Lamparter 2019). Er weist auf die damit verbundene „Vergeudung“ von Ressourcen, aber auch die geringeren Chancen der Älteren, am Arbeits- und Erwerbsleben teilzunehmen, hin.

Zwar ist die Anzahl der älteren Beschäftigten in den letzten Jahren deutlich gewachsen und die Verantwortlichen in den Betrieben haben ihre Aufmerksamkeit auch vermehrt auf die Chancen und Ansatzpunkte einer alters- und alternsgerechten Arbeitsgestaltung gelenkt, jedoch zeigen wissenschaftliche Studien z.B. auf der Basis des IAB-Betriebspanel, dass die betrieblichen Aktivitäten zur Erhaltung der Arbeitsfähigkeit ihrer Beschäftigten nur geringfügig zugenommen haben (Bellmann et al. 2018b). Vor allem in mittelständischen Betrieben müsste mehr getan werden, um den Herausforderungen des wirtschaftlichen und vor allem des demografischen Wandels gerecht zu werden (Bellmann et al. 2018a). Auch die hohen Arbeitsbelastungen sowie die beschränkten Möglichkeiten der (Weiter-)Beschäftigung führen oftmals zu einem früheren Renteneintritt - auch weil für viele Arbeitnehmer*innen nach dem Auslaufen der staatlichen Förderung der Altersteilzeit entsprechende Anreize weiterbestehen.

In meinem Beitrag werden zunächst die Entwicklung der Beschäftigung von Älteren und die Herausforderungen des demografischen Wandels skizziert, der dazu führt, dass in Deutschland mehr Ältere länger arbeiten. Im Abschnitt 3 wird die Situation der älteren Arbeitslosen gesondert betrachtet. Der Abschnitt 4 ist der Verbreitung betrieblicher Maßnahmen zur Erhaltung der Arbeits- und Beschäftigungsfähigkeit Älterer gewidmet. Der Abschnitt 5 enthält das Fazit. 


\section{Entwicklung der Beschäftigung Älterer und der demografische Wandel}

Die Erwerbstätigkeit der Älteren (55- bis unter 65-Jährigen) hat sich nach den Berechnungen des Statistischen Bundesamts (2019) im Zeitraum 1995 bis 2018 stark erhöht. Die Abbildung 1 zeigt, dass der Anteil der älteren Männer, die sich in einem Beschäftigungsverhältnis befinden, in diesem Zeitraum von $48 \%$ auf $76 \%$ gestiegen ist. Bei den Frauen hat sich die entsprechende Quote von $27 \%$ auf $67 \%$ sogar mehr als verdoppelt. Auch haben sich die Erwerbsquoten von Frauen und Männern angenähert: Der Abstand zwischen der Erwerbsquote der Männer und der der Frauen ist von 21 auf 9 Prozentpunkte deutlich gesunken.

\section{Abbildung 1: Erwerbstätigenquote Älterer (55-bis unter 65-Jäbrige)}

1995-2018, in Prozent

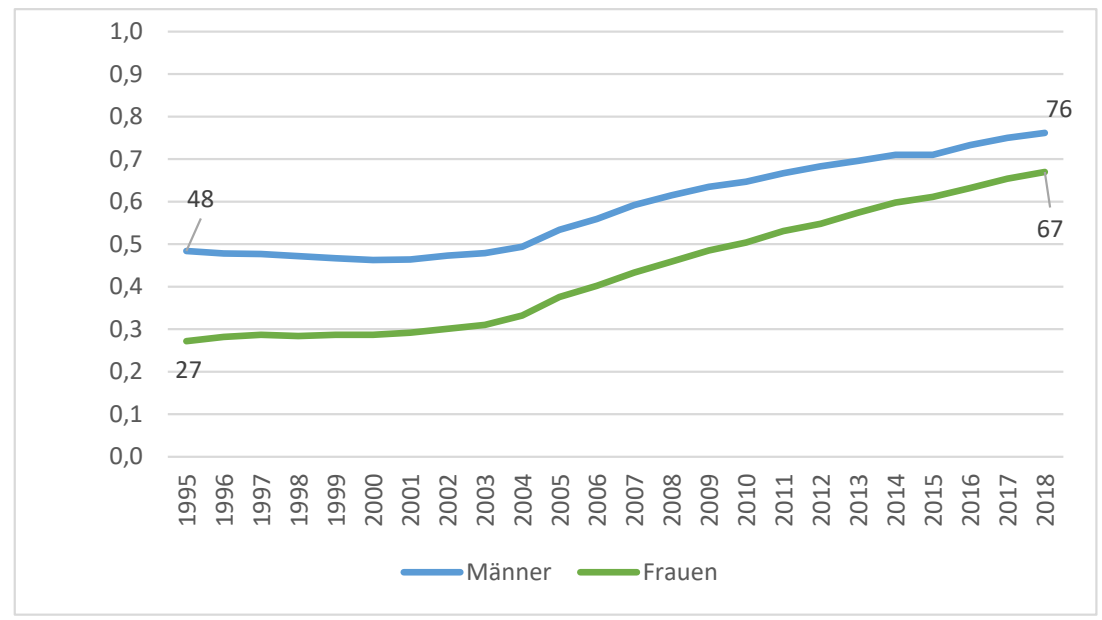

Quelle: Statistisches Bundesamt (2019)

Hinsichtlich der Erwerbstätigenquote der Älteren (50 und älter) im Vergleich zur Erwerbstätigenquote der mittleren Altersgruppe (30 bis 54 Jahre) bestehen nach den Berechnungen von Walwei (2019) auf der Basis des Mikrozensus aber nach wie vor erhebliche Unterschiede: Aus Abbildung 2 geht eine Erwerbsquote der Älteren von $66 \%$ hervor, ab der mittleren Altersgruppen von $84 \%$. Für die Personen mit abgeschlossener Berufsausbildung ergeben sich fast dieselben Werte, während für Hochschulabsolventen höhere Erwerbsquoten aber geringere Abstände zwischen den Älteren und der mittleren Altersgruppe ermittelt werden. Im Unterschied dazu 
sind die Erwerbstätigenquoten der nicht-formal Qualifizierten niedriger und die Unterschiede zwischen den Altersgruppen vergleichsweise geringer ausgeprägt.

\section{Abbildung 2: Erwerbstätigenquote nach Qualifikation und Alter 2017, in Pro- zent}

\section{Erwerbstätigenquote nach Qualifikation und Alter 2017, in Prozent}

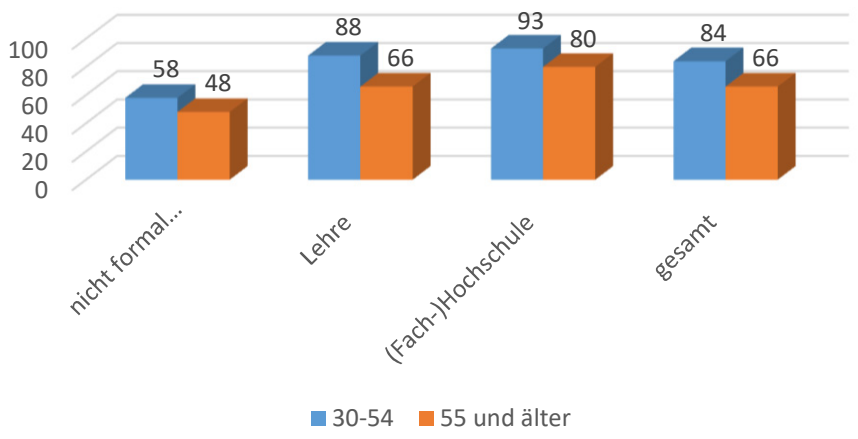

Quelle: Walwei (2019), Berechnungen auf Basis des Mikrozensus 2017

Allerdings muss beim Vergleich der Altersgruppen auch berücksichtigt werden, dass bei den abhängig Beschäftigten, die nach Erreichen der gesetzlichen Altersgrenze weiterarbeiten, der Anteil der Vollzeitbeschäftigten je nach Geburtskohorte zwischen 2 und $6 \%$ bei den Frauen und zwischen 8 und $15 \%$ bei den Männern variiert. Der Anteil der in Teilzeit beschäftigten Männer und Frauen liegt in einer ähnlichen Größenordnung, während die geringfügig Beschäftigten in der Mehrheit sind (Westermeier 2019).

Die Entwicklung des gesamtwirtschaftlichen Arbeitskräfteangebots wird nicht nur von den Erwerbstätigenquoten, sondern auch von der Migration und der Entwicklung der nachwachsenden Kohorten Jüngerer und dem altersbedingten Ausscheiden bestimmt. Möller/Walwei (2013) weisen darauf hin, dass ein demografisch bedingt niedrigeres Arbeitskräfteangebot dauerhaft den Kapitalstock und damit das Wachstumspotenzial einer Volkswirtschaft reduziert.

In den letzten Jahren wurde nach den IAB-Projektionen der demografische Effekt zusätzlich durch die große Zuwanderung reduziert (Fuchs et al. 2017). Grund dafür waren jedoch eher singuläre Entwicklungen wie 
eine in den letzten Jahren verstärkte Migration aus anderen EU-Staaten nach Deutschland und die stake Fluchtmigration besonders im Jahr 2015.

Darüber hinaus ist das Arbeitsangebot seit Längerem einem Alterungsprozess unterworfen, so dass dem Arbeitsangebot älterer Erwerbspersonen eine wachsende Bedeutung zukommt. Fuchs et al. (2011) haben einerseits eine Verringerung der Anzahl von verfügbaren Arbeitskräften in den Altersgruppen zwischen 15 und 29 sowie zwischen 30 und 49 Jahren ermittelt. Auf der anderen Seite wuchs die Anzahl der Erwerbspersonen in den Altersgruppen der 50- bis 74-Jährigen. Dabei wirkt sich noch bis zum Jahr 2030 der Effekt der „Babyboomer-Generation“ aus.

Diese Entwicklungen bedeutet, dass es in den nächsten Jahren aus zwei Gründen darauf ankommen wird, die Älteren verstärkt in Beschäftigung zu integrieren: Erstens, weil diese Gruppe zahlenmäßig größer wird und zweitens, weil damit der Reduktion des Erwerbspersonenpotenzials durch die niedrigeren Erwerbsquoten Älterer entgegengewirkt werden kann.

\section{2. Ältere Arbeitslose}

Wie bereits erwähnt, wird die Erwerbstätigkeit von Arbeitskräften relativ stark von ihrer Qualifikation bestimmt, wobei Personen ohne formalen Berufsabschluss die niedrigsten Erwerbsquoten aufweisen: Dies trifft in besonderer Weise für die Älteren zu. Aufgrund einer Sonderregelung im Sozialgesetzbuch II gelten Erwerbsfähige nach Vollendung des 58. Lebensjahres nicht mehr als arbeitslos, wenn sie zuvor für mindestens zwölf Monate Grundsicherung für Arbeitsuchende bezogen haben und ihnen keine sozialversicherungspflichtige Beschäftigung angeboten worden ist. Während 2008 die Anzahl der Personen, die unter diese Regelung fielen, noch bei knapp 23.000 lag, waren es 2019 mit 171.276 mehr als siebenmal so viele. Damit werden für die Arbeitsagenturen Anreize geschaffen, sich nicht mehr durch arbeitsmarktpolitische Maßnahmen um eine Integration der Angehörigen dieser Gruppe in ein Beschäftigungsverhältnis zu kümmern.

Darüber hinaus ist das letzte Drittel des Erwerbslebens besonders wichtig bei der Berechnung der Ansprüche an die gesetzliche Rentenversicherung. Längere Dauer der Arbeitslosigkeit während dieses Zeitraums und ein vorzeitiger Eintritt in den Ruhestand reduzieren die Höhe der zu erwartenden Rente (Kaboth/Brussig 2020).

Die Betrachtung von Arbeitsmarktrisiken anhand der Arbeitslosigkeit in Abbildung 3 zeigt, dass die Arbeitslosenquote insgesamt deutlich nach dem Ende der Großen Rezession 2008/2009 gesunken ist, wobei sie in der Großen Rezession bei den Älteren von 7,9\% auf 8,7\% wesentlich stärker 
als die Arbeitslosenquote insgesamt gestiegen ist. Insofern ist es nicht überraschend, dass die Anzahl der Arbeitslosen seit 2009 zwar von 3,4 Millionen auf 2,3 Millionen im Jahr 2018 um fast ein Drittel zurückgegangen ist, aber der Anteil der älteren Arbeitslosen von $15 \%$ im Jahr 2009 auf $21 \%$ im Jahr 2018 deutlich gestiegen ist.

\section{Abbildung 3: Arbeitslose und Arbeitslosenquoten von Älteren (55 bis unter 65) und insgesamt}

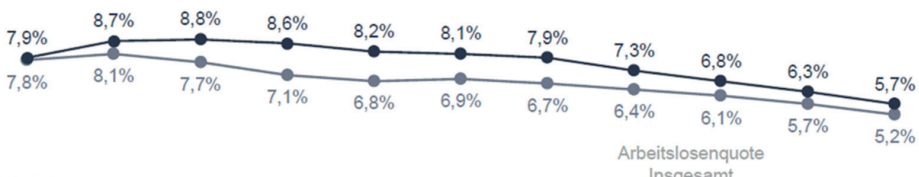

Arbeitslose

15 bis unter 65 Jahre in Millionen

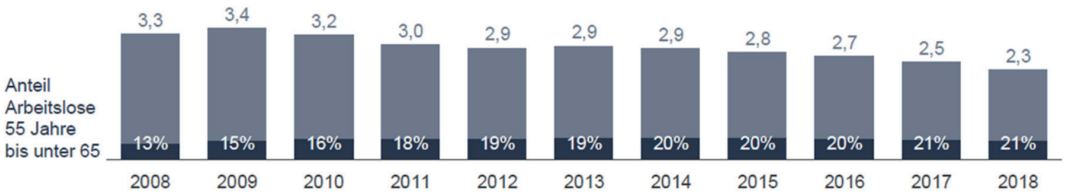

Quelle: Bundesagentur für Arbeit (2019)

Grundsätzlich wird die Dynamik der Arbeitslosigkeit vom Risiko des Zugangs in und die Chancen des Abgangs aus der Arbeitslosigkeit bestimmt. Studien der Bundesagentur für Arbeit (2017) zeigen zwar, dass erfreulicherweise für die Älteren im Zeitraum Dezember 2014 bis Dezember 2018 das Zugangsrisiko von 0,56 auf 0,46\% gesunken ist, während sich die Abgangschancen von 3,06 auf 3,64\% erhöht haben, allerdings sind die $\mathrm{Zu}$ gangsrisiken der Älteren größer und vor allem ihre Abgangschancen sehr viel geringer als die der anderen Arbeitslosen.

Auf der Basis einer Stichprobe der Erwerbspersonen haben Homrighausen/Wolf (2018) die Wiederbeschäftigungschancen von Arbeitslosen verschiedener Altersgruppen untersucht. Aus der Abbildung 4 geht hervor, dass $79 \%$ aller Frauen in der Altersgruppe von 47 bis 49 Jahren und $80 \%$ aller Männer innerhalb von zwei Jahren nach Beginn ihres Arbeitslosengeldbezugs wieder eine sozialversicherungspflichtige Beschäftigung aufnehmen konnten. Die älteren Arbeitslosen haben aber wesentlich geringere Wiederbeschäftigungschancen: Bei den Frauen sinken die Chancen für die 58 - bis 60 -Jährigen auf nur noch $27 \%$, für 61- bis 62-Jährige auf $11 \%$ und für 63- bis 64-Jährige auf $5 \%$. Bei den Männern sieht es etwas besser aus: Ihre Chancen als 58- bis 60-jähriger Arbeitsloser eine sozialversiche- 
rungspflichtige Beschäftigung aufzunehmen, betragen $42 \%$, als 61- bis 62Jähriger $16 \%$ und für 63- bis 64-Jähriger $9 \%$. Abbildung 4 zeigt den Anteil der Arbeitslosen, die innerhalb der ersten zwei Jahre nach Beginn des Arbeitslosengeldbezugs erneut sozialversicherungspflichtig beschäftigt waren, nach Geschlecht und Altersgruppen, in Prozent.

\section{Abbildung 4: Wiederbeschäftigungschancen Älterer, in Prozent}

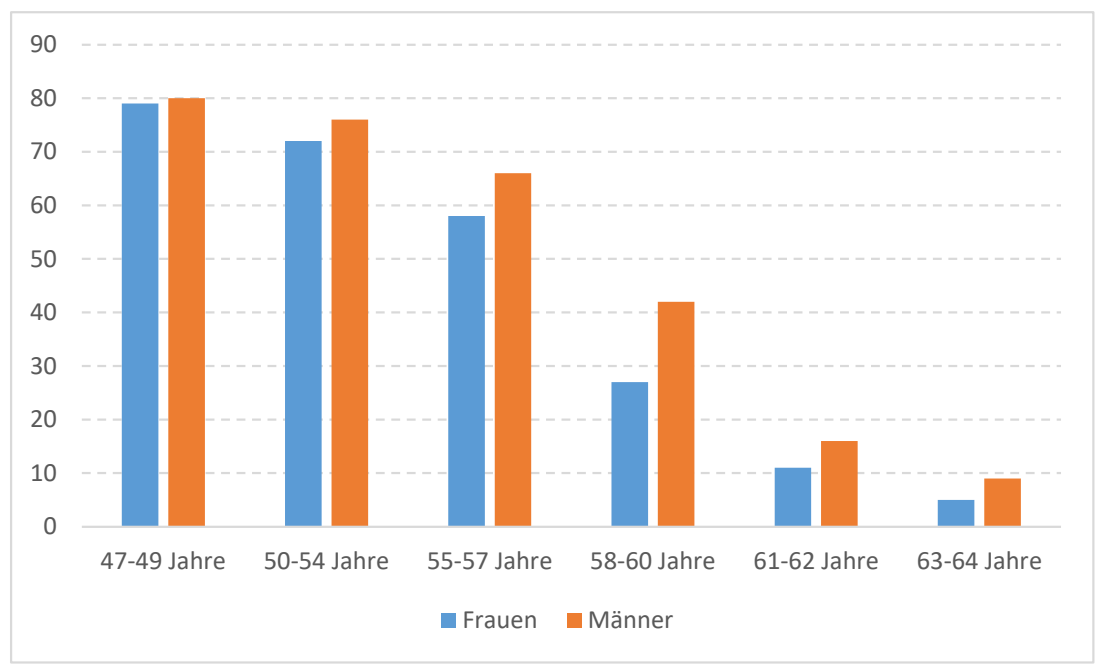

Quelle: Homrighausen/Wolf (2018)

Die im Rahmen der Studie von Homrighausen/Wolf (2018) im Jahre 2016 durchgeführte Befragung von 63 Vermittlungsfachkräften in den Agenturen für Arbeit zum Vermittlungsprozess arbeitsloser Personen ab 55 Jahren ergab, dass diese Menschen besondere Schwierigkeiten haben, eine neue Beschäftigung zu finden. Die Tabelle 1 zeigt, dass gesundheitliche Einschränkungen und aktuell zu geringe oder veraltete EDV-Kenntnisse die am häufigsten genannten Hemmnisse waren. Auch grundsätzliche Vorbehalte von Unternehmen gegenüber älteren Arbeitskräften sind für $70 \%$ der befragten Vermittlungsfachkräfte ein typisches Vermittlungshemmnis Älterer. Vor allem bei älteren Frauen werden die eingeschränkte Mobilität und Einschränkung durch familiäre Verpflichtungen wie Betreuung und Pflege von Angehörigen genannt. Bei den Männern wird (aber nicht signifikant) häufiger als bei Frauen auf zu hohe Lohnansprüche Älterer hingewiesen. 
Tabelle 1: Spezifische Vermittlungshemmnisse bei Personen ab 55 Jahren aus Sicht der Vermittlungsfachkräfte, in Prozent

\begin{tabular}{|l|c|c|}
\hline Vermittlungshemmnisse bei Älteren & Frauen & Männer \\
\hline gesundheitliche Einschränkungen vorhanden & 89 & 90 \\
\hline EDV-Kenntnisse zu gering/veraltet & 89 & 80 \\
\hline $\begin{array}{l}\text { Vorbehalte jeglicher Art bei Unternehmen ge- } \\
\text { genüber Älteren }\end{array}$ & 70 & 70 \\
\hline örtliche Mobilität eingeschränkt & 74 & 52 \\
\hline $\begin{array}{l}\text { Einschränkung durch familiäre Verpflichtun- } \\
\text { gen (Betreuung/Pflege/Sonstiges) }\end{array}$ & $67^{* *}$ & 20 \\
\hline $\begin{array}{l}\text { Lohnanspruch Älterer zu hoch } \\
\text { Fremdsprachenkenntnisse zu gering/veraltet }\end{array}$ & 54 & 54 \\
\hline $\begin{array}{l}\text { Motivation zur Lern-/Weiterbildungsbereit- } \\
\text { schaft eingeschränkt }\end{array}$ & 51 & 56 \\
\hline $\begin{array}{l}\text { Berufliche Qualifikation (Abschluss/Anerken- } \\
\text { nung) fehlt oder veraltet }\end{array}$ & 52 & 49 \\
\hline $\begin{array}{l}\text { Motivation zur Eigeninitiative/Arbeitshaltung } \\
\text { eingeschränkt }\end{array}$ & 44 & 16 \\
\hline Berufserfahrung fehlt oder veraltet & 30 & 46 \\
\hline
\end{tabular}

* signifikante Unterschiede zwischen den Angaben der Befragten für beide Geschlechter auf dem $5 \%$-Niveau

Quelle: Homrighausen/Wolf (2018)

\section{Erhaltung der Arbeits- und Beschäftigungsfähigkeit Älterer}

Die vorgetragenen Argumente zur Notwendigkeit und Bedeutung der Integration der Älteren in Beschäftigung werfen die Frage auf, unter welchen Voraussetzungen Ältere einen Arbeitsplatz finden bzw. länger in Beschäftigung bleiben. Besondere Bedeutung hat in diesem Kontext das Konzept der Arbeits- und Beschäftigungsfähigkeit erlangt, wobei beide Begriffe in der Literatur wie in der öffentlichen Diskussion uneinheitlich verwendet werden.

Ilmarinen (2006) sieht in der Arbeitsfähigkeit das Zusammenspiel der persönlichen Fähig- und Fertigkeiten mit den Anforderungen des Arbeitsmarktes. Die physische und psychische Gesundheit, Kompetenzen, Motivation, Einstellungen, arbeitsplatzbezogene Anforderungen und Belastungen sind für die Arbeitsfähigkeit zentral. Ansatzpunkte für die Aufrechter- 
haltung der Kompetenzen, Gesundheit und Motivation ergeben sich deshalb beispielsweise in den Bereichen Bildung, Arbeitsplatz- und Arbeitszeitgestaltung, Arbeitsorganisation, Gesundheitsschutz, Laufbahngestaltung und Unternehmenskultur.

Die Ergebnisse des IAB-Betriebspanels in Tabelle 2 zeigen, dass der Anteil der Betriebe, die für ältere Beschäftigte Maßnahmen zum Erhalt ihrer Arbeits- und Beschäftigungsfähigkeit anbieten, eher gering und im Zeitablauf sogar leicht gesunken ist (Bellmann et al. 2018a, 2018b).

Tabelle 2: Anteil der Betriebe mit Maßnahmen für Ältere Beschäftigte im Zeitverlauf, in Prozent

\begin{tabular}{|l|c|c|c|c|}
\hline & $\mathbf{2 0 0 6}$ & $\mathbf{2 0 0 8}$ & $\mathbf{2 0 1 1}$ & $\mathbf{2 0 1 5}$ \\
\hline Altersteilzeit & 10 & 9 & 8 & 5 \\
\hline $\begin{array}{l}\text { Besondere Ausstattung der Arbeitsplät- } \\
\text { ze }\end{array}$ & 1 & 2 & 2 & 3 \\
\hline $\begin{array}{l}\text { Individuelle Anpassung der Leistungs-/ } \\
\text { Arbeitsanforderungen }\end{array}$ & 2 & 2 & 4 & 5 \\
\hline $\begin{array}{l}\text { Altersgemischte Besetzung von Ar- } \\
\text { beitsgruppen }\end{array}$ & 5 & 5 & 6 & 5 \\
\hline $\begin{array}{l}\text { Einbeziehung Älterer in betriebliche } \\
\text { Weiterbildung }\end{array}$ & 6 & 6 & 9 & 7 \\
\hline Spezielle Weiterbildung für Ältere & 1 & 1 & 1 & 1 \\
\hline $\begin{array}{l}\text { Einbeziehung Älterer in Maßnahmen } \\
\text { zur Gesundheitsförderung }\end{array}$ & - & - & 4 & 4 \\
\hline Andere Maßnahmen für Ältere & 1 & 1 & 1 & 1 \\
\hline
\end{tabular}

Quelle: Bellmann et al. (2018a, 2018b), Berechnungen auf der Basis des IAB-Betriebspanels.

In der Erhebung 2015 erwies sich mit $7 \%$ der Nennungen die betriebliche Weiterbildung als das wichtigste Instrument, gefolgt von der besonderen Ausstattung des Arbeitsplatzes ( $5 \%$ der Nennungen) sowie der individuellen Anpassung der Leistungs- bzw. Arbeitsanforderungen (ebenfalls $5 \%$ der Nennungen) und der Altersteilzeit (ebenfalls $5 \%$ der Nennungen). Allerdings wird die Altersteilzeit in den weitaus meisten Fällen im Blockmodell realisiert, was eben nicht der (Weiter-)Beschäftigung bis zur Altersgrenze, sondern einem vorzeitigen Übergang in den Ruhestand dient und mit einem (vorzeitigen) Ausscheiden aus dem Erwerbsleben verbunden ist. 
Damit lässt sich ein klares Defizit bei der Verbreitung von betrieblichen Maßnahmen zur Förderung der Arbeits- und Beschäftigungsfähigkeit Älterer konstatieren.

\section{Abbildung 5: Anteil der Betriebe mit Maßnabmen für ältere Beschäftigte (50+)} nach Betriebsgröße 2015, in Prozent

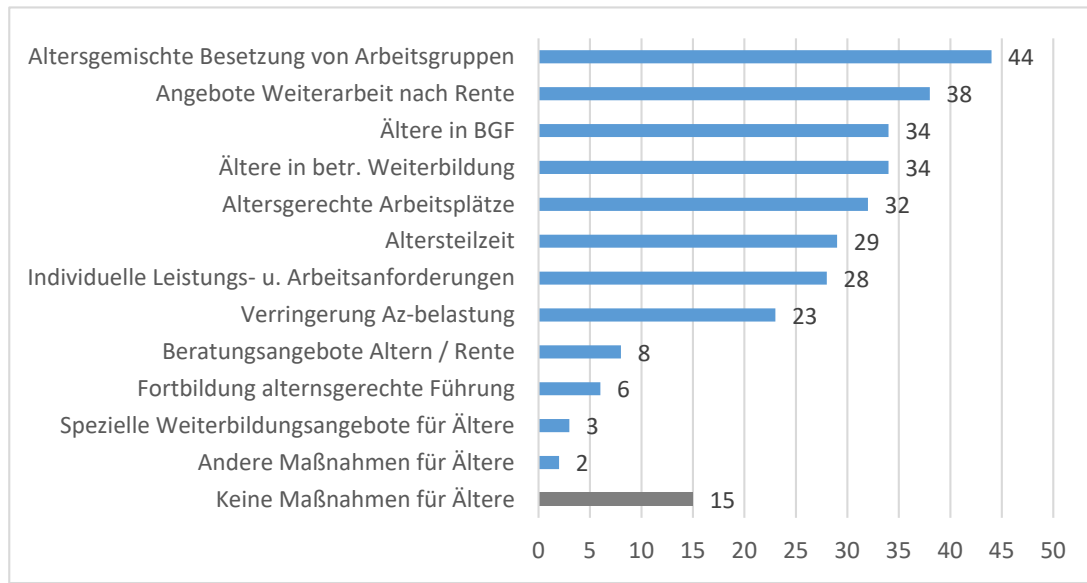

Basis: Betriebe mit älteren (über 50-jährigen) Beschäftigten

Quelle: Bellmann et al. (2018a, 2018b) auf der Basis des IAB-Betriebspanels 2015

Aus der Abbildung 5 geht hervor, dass der Grad der Verbreitung dieser Maßnahmen in Betrieben mit 500 und mehr Beschäftigten bei $87 \%$ liegt, aber schon in der Größenklasse von 100 bis 499 Beschäftigten mit $65 \%$ wesentlich geringer ist. In kleineren und mittleren Betrieben reduziert sich der Anteil der Betriebe mit wenigstens einer Maßnahme von den in der Tabelle 2 genannten Maßnahmen zu Erhalt ihrer Arbeits- und Beschäftigungsfähigkeit dann weiter. Damit wird deutlich, dass die Betriebsgröße einen wesentlichen Einfluss ausübt.

In dem von der Hans-Böckler-Stiftung finanzierten Projekt „Einstellung und Weiterbeschäftigung älterer Arbeitnehmer in Betrieben der Chemieindustrie (EBA)“ wurde im Sommer 2016 eine Gruppe von 385 kleinen und mittleren Betrieben (mit 20 bis 500 Beschäftigten) der chemischen Industrie zu verschiedenen Aspekten des Umgangs mit älteren Arbeitnehmer*innen online befragt. Die Chemiebranche ist deshalb besonders interessant, weil hier auf die Demografie bezogene Tarifvereinbarungen eine relativ große Rolle spielen, so dass davon auszugehen ist, dass die Altersdiskriminierung (kaum) eine Rolle spielt. Vorliegende branchenspezifische 
Auswertungen des IAB-Betriebspanels bestätigen diese Aussage (Bellmann et al. 2012).

Nach den Ergebnissen unserer Studie bieten mehr als acht von zehn Betrieben ihren älteren Beschäftigten und Führungskräften mindestens eine betriebliche Maßnahme zur Erhaltung ihrer Arbeits- und Beschäftigungsfähigkeit an. In der Abbildung 6 sind die betrieblichen Maßnahmen dargestellt, die von den Betrieben häufiger und weniger häufig genutzt werden: eine altersgemischte Besetzung von Arbeitsgruppen (44\%), Angebote, nach dem Renteneintritt weiter für den Betrieb tätig zu bleiben (38\%) sowie die Einbeziehung Älterer in Maßnahmen zur Gesundheitsförderung und in die Weiterbildungsaktivität (jeweils $34 \%$ ). Interessant ist auch die vergleichsweise geringe Reduzierung von Arbeitszeitbelastungen (23\%); möglicherweise hängt dies aber auch mit den besonderen tarifvertraglichen Möglichkeiten zusammen.

Abbildung 6: Betriebliche Maßnahmen für ältere Beschäftigte, in Prozent

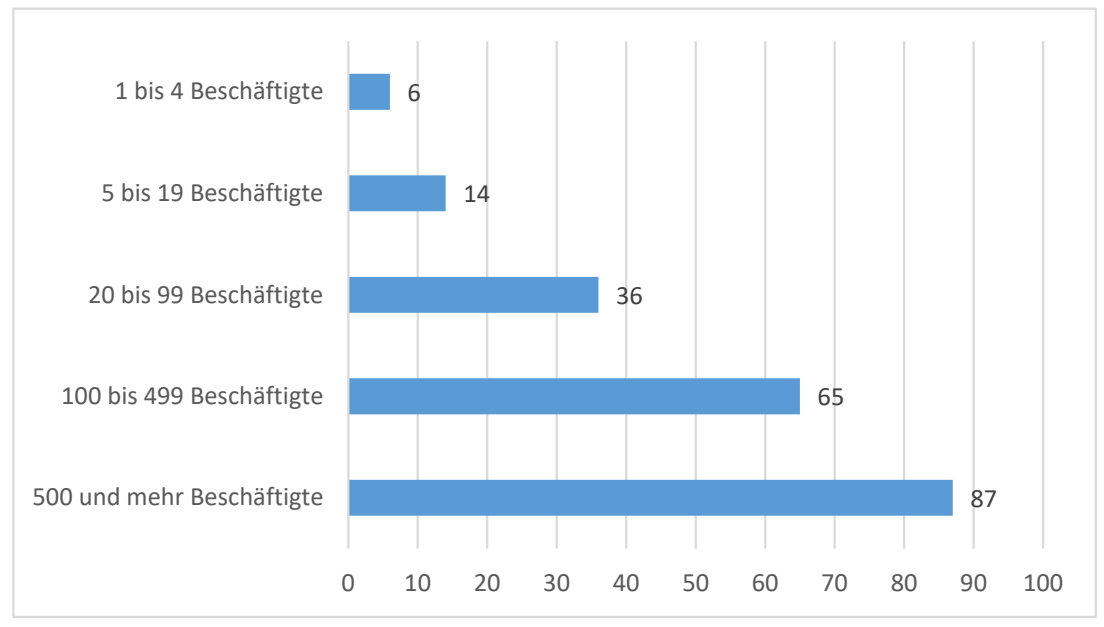

Quelle: Bellmann et al. (2018a, 2018b) auf Basis der EBA-Branchenbefragung 2016

Insgesamt sind vor allem die Ergebnisse zur Weiterbildungsbeteiligung enttäuschend, gerade vor dem Hintergrund der zunehmenden Bedeutung der Notwendigkeit der Weiterentwicklung der Fähigkeiten und Kompetenzen in Verbindung mit der Digitalisierung, wie sie von jüngeren und älteren Beschäftigten gleichermaßen in der Erhebung des Linked Personnel Panel 2015 berichtet wurden (Bellmann 2017). Hier sind Steigerungen der Weiterbildungsbeteiligung der Älteren erforderlich. 
Wie groß das Defizit noch ist, wird auch aus den Daten des IAB-Betriebspanels ersichtlich. Abbildung 7 ist zu entnehmen, dass seit 2010 sowohl der Anteil der Weiterbildungsteilnehmer"innen an den Beschäftigten als auch der Anteil der weiterbildenden Betriebe an allen Betrieben deutlich zugenommen hat: Der Anteil der Weiterbildungsteilnehmer*innen ist von $18 \%$ im Jahr 2001 auf $35 \%$ im Jahr 2017 gestiegen. Der Anteil der weiterbildenden Betriebe hat von $36 \%$ auf $53 \%$ im selben Zeitraum zugenommen. Mit dem zuletzt genannten Anteil lässt sich der Anteil der Betriebe, die ihre älteren Mitarbeiter*innen in die betriebliche Weiterbildung einbeziehen, durchaus vergleichen. Nach Tabelle 2 beläuft sich dieser Wert aber nur auf $7 \%$.

\section{Abbildung 7: Betriebliche Weiterbildungsaktivitäten}

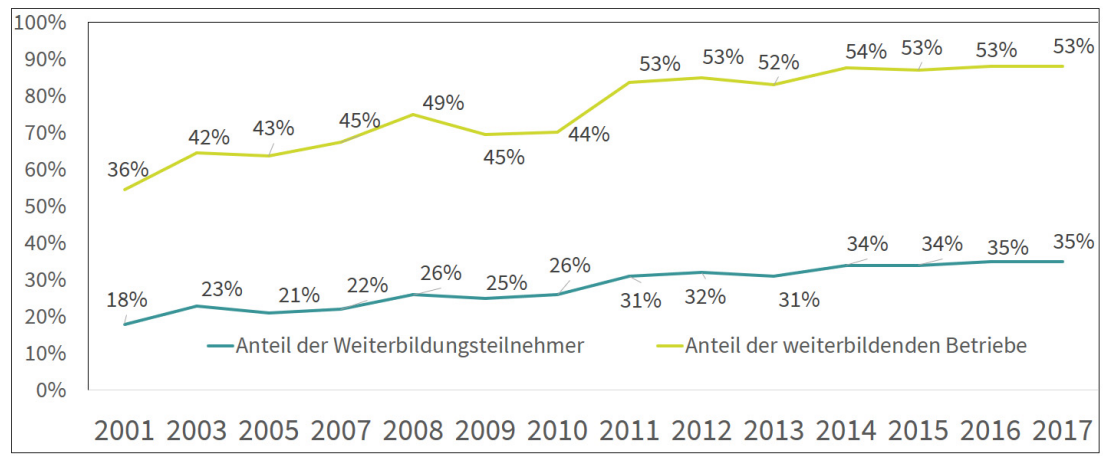

Quelle: eigene Berechnungen mit den Daten des IAB-Betriebspanels

\section{Fazit}

In diesem Beitrag konnte gezeigt werden, dass sich die Erwerbsbeteiligung Älterer im Zeitraum von 1995 bis 2018 deutlich erhöht hat. Dies liegt auch an der zunehmenden Erwerbsbeteiligung der Frauen. Ältere Beschäftigte verbleiben immer länger in den Betrieben, in denen sie bereits länger beschäftigt sind. Dagegen tun sich Ältere, die einen neuen Arbeitsplatz suchen, nach wie vor schwer, einen neuen Arbeitsplatz zu finden. Dies gilt vor allem für ältere Arbeitslose.

Obwohl die Aufrechterhaltung der Kompetenzen, Gesundheit und Motivation sowie die Reduktion arbeitsplatzbezogener Anforderungen und Belastungen für die Erhaltung der Arbeits- und Beschäftigungsfähigkeit Älterer anerkannt sind, ist die Verbreitung entsprechender betrieblicher 
Maßnahmen nach wie vor gering. Trotz der zunehmenden Bedeutung der Notwendigkeit der Weiterentwicklung von Fähigkeiten und Kompetenzen in der modernen Arbeitswelt für alle Altersgruppen, bestehen Defizite vor allem bei kleineren und mittleren Betrieben.

Diese Entwicklungen stehen im Widerspruch zur Notwendigkeit der Integration der Älteren in die Beschäftigung, deren zunehmende Erwerbsbeteiligung den Rückgang des Erwerbspersonenpotenzials bei wieder sinkender Migration, kleineren Geburtskohorten und des Übergangs der „Babyboomer-Generation" in den Ruhestand kompensieren müsste.

\section{Literatur}

Adamy, W., Breutmann, N., Hinz, A., Horst, A., Richter, G., Serries, Chr., Schmander, M. (2017): Übergreifende Aspekte einer alters- und alternsgerechten Arbeitsgestaltung. In: G. Richter, Chr. Hecker, A. Hinz (Hrsg.): Produktionsarbeit in Deutschland - mit alternden Belegschaften. Berlin: Erich Schmidt Verlag, $42-55$.

Bellmann, L. (2017): Chancen und Risiken der Digitalisierung für ältere Produktionsarbeiter. IAB-Forschungsbericht 15/2017, Nürnberg.

Bellmann, L., Brandl, S., Dummert, S., Guggemos, P., Leber, U., Matuschek, I. (2018a): Altern in Betrieb. Alterung und Alter(n)smanagement in kleineren und mittleren Unternehmen - vom Einzelfall zur professionalisierten Systematik (Hans-Böckler-Stiftung. Study, 393), Düsseldorf.

Bellmann, L., Dummert, S., Leber, U. (2018b): Konstanz altersgerechter Maßnahmen trotz steigender Beschäftigung Älterer. WSI-Mitteilungen 71(1), 20-27.

Bellmann, L., Leber, U., Möller, I. (2012): Fachkräftebedarf in ausgewählten Branchen aus dem Organisationsbereich der IG BCE, Hannover.

Bundesagentur für Arbeit (2017): Arbeitsmarktberichte. Nürnberg.

Bundesagentur für Arbeit (2019): Arbeitsmarktberichte. Nürnberg.

Czepek, J., Moczall, A.: (2017): Neueinstellung Älterer: Betriebe machen meist gute Erfahrungen. IAB-Kurzbericht 08/2017, Nürnberg.

Czepek, J., Gürtzgen, N., Moczall, A., Weber, E. (2017): Halten rentenberechtigter Mitarbeiter in den Betrieben: Vor allem kürzere und flexiblere Arbeitszeiten kommen zum Einsatz. IAB-Kurzbericht 16/2017, Nürnberg.

Fuchs, J., Hummel, M., Hutter, C., Klinger, S., Wanger, S., Weber, E., Zika, G. (2017): IAB-Prognose 2017: Der Arbeitsmarkt stellt neue Rekorde auf. IAB-Kurzbericht 09/2017, Nürnberg.

Fuchs, J., Söhnlein, D., Weber, B. (2011): Projektion des Erwerbspersonenpotenzials bis 2050: Rückgang und Alterung sind nicht mehr aufzuhalten. IAB-Kurzbericht 16/2011, Nürnberg. 
Fuchs, J., Söhnlein, D., Weber, B. (2017): Projektion des Erwerbspersonenpotenzials bis 2060: Arbeitskräfteangebot sinkt auch bei hoher Zuwanderung. IABKurzbericht 06/2017, Nürnberg.

Homrighausen, P., Wolf, K. (2018): Wiederbeschäftigungschancen Älterer: Wo Vermittlungskräfte Handlungsbedarf sehen. IAB-Kurzbericht 11/2018, Nürnberg.

Ilmarinen, J. (2006): Towards a longer Worklife: Ageing and the Quality of Worklife in the European Union, Helsinki: Finish Institute of Occupational Health.

Kaboth, A., Brussig, M. (2020): Trotz Alterserwerbsbeteiligung auf Rekordniveau: Mehr Ältere von Arbeitslosigkeit betroffen. Institut Arbeit und Qualifizierung (IAQ).

Lamparter, D. (2019): Die Vergeudung. Die Zeit vom 18. Dezember 2019, 30.

Möller, J., Walwei, U. (2013): Konsequenzen des demografischen Wandels für die Arbeitswelt: Ende der Arbeitslosigkeit nicht in Sicht? In: H. Hinte, K. F. Zimmermann (Hrsg.): Zeitenwende auf dem Arbeitsmarkt. Wie der demografische Wandel die Erwerbsgesellschaft verändert, Bonn: Bundeszentrale für politische Bildung, 114-141.

Walwei, U. (2018): Trends in der Beschäftigung Älterer: Rahmenbedingungen für betriebliche Personalpolitik. WSI-Mitteilungen 71(1), 3-11.

Westermeier, Chr. (2019): Eine stabile Beschäftigung vor dem Rentenalter begünstigt die Weiterarbeit. IAB-Kurzbericht 15/2019, Nürnberg. 
